\title{
¿Los ecuatorianos con asma tienen preferencias en el uso de tecnologías de la información y comunicación? Estudio piloto
}

\author{
Iván Chérrez-Ojeda, ${ }^{1,2}$ Karin Plaza, ${ }^{1,2}$ José A. Cano, ${ }^{1,2}$ Juan Carlos Calderón, ${ }^{1,2}$ Annia Chérrez, ${ }^{1,6}$ Alan Baptist, ${ }^{3}$ \\ Erick Calero, ${ }^{1,2}$ Rodrigo Hoyos, ${ }^{4}$ Patricio Beltrán, ${ }^{5}$ Víctor Cruz, ${ }^{7}$ Germán Ramón, ${ }^{8}$ Daniel Simancas ${ }^{9}$
}

\begin{abstract}
Background: Inadequate communication between asthmatic patients and their physicians may interfere directly with asthma control. In the last years, the use of information and communication technologies (ICTs) it has increased in Latin-America. This technology seems to be a good tool to improve communication and management of the asthmatic patient.

Objective: We evaluated the frequency and preference patterns of communication and information technologies in Ecuadorian patients with bronchial asthma.

Methods: We conducted a pilot cross-sectional study to identify the frequency and preferences of ICT in patients with asthma. The Spanish version of the Michigan questionnaire was used. Age and educational level were categorized into 3 groups. We used logistic regression between these groups regarding the frequency of use, interest in seeking and receiving information related to their asthma for the age and educational level of the patients.

Results: A total of 222 patients participated in our study. The mean age was 45.6 years (SD 17.4), the most common sex was female with $89.25 \%$. Almost all patients had a cell phone $(87.5 \%)$ and internet access $(62.7 \%)$. The three ICTs most likely to be used to search for or receive information about their illness were WhatsApp, Facebook and email $(p<0.05)$.

Conclusion: Information and communication technologies improve the care of asthmatic patients. In our pilot study email and text messages are the most preferred ICT among patients however WhatsApp and Facebook may be appropriate for certain ages especially young asthmatic. Knowledge of preferences can help the development of ICT in a personalized way and improve the outcomes in patients with asthma.
\end{abstract}

Keywords: Asthma; Social media; Information and communication technologies; Internet

Este artículo debe citarse como: Chérrez-Ojeda I, Plaza K, Cano JA, Calderón JC, Chérrez A, Baptist A, Calero E, Hoyos R, Beltrán P, Cruz V, Ramón G, Simancas D. ¿Los ecuatorianos con asma tienen preferencias en el uso de tecnologías de la información y comunicación? Estudio piloto. Rev Alerg Mex. 2017;64(4):403-414

${ }^{1}$ Respiralab, Research Group. Guayaquil, Ecuador.

2Universidad Espíritu Santo. Samborondón, Guayas, Ecuador ${ }^{3}$ Universidad de Michigan, Departamento de Medicina Interna. Ann Arbor, Michigan, Estados Unidos

${ }^{4}$ Hospital de los Valles. Quito, Ecuador

${ }^{5}$ Instituto Ecuatoriano de Seguridad Social, Hospital Portoviejo,

Departamento de Neumología. Portoviejo, Manabí, Ecuador

\author{
${ }^{6}$ Universidad de Heidelberg, Escuela de Medicina. Heidelberg, \\ Baden-Wurtemberg, Alemania \\ ${ }^{7}$ Ministerio de Salud Pública, Hospital Neumológico "Dr. Alfredo J. \\ Valenzuela". Guayas, Guayaquil, Ecuador \\ ${ }^{8}$ Hospital Italiano Regional del Sur, Sección Alergia. Bahía Blanca, \\ Buenos Aires, Argentina
}




\section{Resumen}

Antecedentes: La comunicación inadecuada entre los pacientes y sus médicos puede interferir directamente con el control del asma. En los últimos años, el uso de tecnologías de la información y comunicación se ha incrementado en Latinoamérica y parece ser un recurso útil para mejorar la comunicación, adherencia y control del paciente con asma.

Objetivo: evaluar la frecuencia y patrones de preferencia de las tecnologías de comunicación e información en pacientes ecuatorianos con asma bronquial.

Métodos: estudio transversal en el que se utilizó la versión en español del cuestionario Michigan. La edad y el nivel educacional fueron categorizados en tres grupos. Se utilizó regresión logística entre los grupos respecto a la frecuencia de uso e interés en buscar y recibir información relacionada con el asma, según la edad y nivel educativo.

Resultados: en nuestro estudio participaron 222 pacientes. La edad media fue de $45.6 \pm 17.4$ años y el sexo más común fue el femenino, con $89.25 \%$. Casi todos los pacientes tenían teléfono celular $(87.5 \%)$ y acceso a internet $(62.7 \%)$. Las tres tecnologías de la información y comunicación con mayor probabilidad de ser usadas con fines de buscar o recibir información acerca de la enfermedad fueron WhatsApp, Facebook y correo electrónico $(p<0.05)$.

Conclusión: el correo electrónico y los mensajes de texto fueron preferidos por los pacientes, sin embargo, WhatsApp y Facebook pueden ser apropiados para ciertos grupos, especialmente para los jóvenes. La selección de TIC adecuadas para la comunicación médico-paciente podría mejorar la adherencia y el control de los pacientes con asma.

Palabras clave: Asma; Medios sociales de comunicación; Tecnologías de información y comunicación; Internet

'Universidad Tecnológica Equinoccial, Facultad de Ciencias de la Salud "Eugenio Espejo", Centro de Investigación en Salud Pública y Epidemiologia Clínica. Quito, Ecuador
Correspondencia: Iván Chérrez-Ojeda.

ivancherrez@gmail.com

Recibido: 2017-05-06

Aceptado: 2017-07-16

\section{Abreviaturas y siglas}

TIC, tecnologías de la información y comunicación

\section{Antecedentes}

El asma en una enfermedad crónica que afecta aproximadamente a $4.3 \%$ de la población mundial. ${ }^{1}$ Asimismo, es una enfermedad que amenaza potencialmente la vida del paciente, con una tasa de mortalidad de 1 por cada 250 casos en el mundo. ${ }^{2}$ Entre los pacientes que la padecen, la prevalencia control parcial o no controlada es de $93 \%$ en Latinoamérica. ${ }^{3}$ Entre los factores que contribuyen al no control del asma se encuentran la falta de adherencia al tratamiento, el mal uso de los inhaladores y la mala comunicación médico-paciente. ${ }^{4}$ Por ejemplo, solo $38 \%$ de pacientes con asma informa el uso diario de su medicación de control. ${ }^{5}$ Por otro lado, las diferencias en las estrategias de comunicación entre médico y paciente también contribuyen. ${ }^{6}$ Por lo anterior, se necesitan nuevas estrategias para mejorar estos factores y lograr una buena comunicación entre los proveedores de salud y los pacientes con asma.

En los últimos 5 años se ha observado un incremento exponencial en el número de usuarios de dispositivos móviles. Más de 2 billones de personas alrededor del mundo usan el internet como fuente principal para la búsqueda de información relacionada con la salud. ${ }^{7}$ Las tecnologías de información y comunicación (TIC) son las que se emplean para 
comunicar, manipular y almacenar datos por medios electrónicos; incluyen correo electrónico, mensajes de texto, video chat y redes sociales, así como todos los diferentes dispositivos que realizan una amplia gama de funciones de comunicación e información. ${ }^{8}$

En la actualidad, resulta de gran interés la posibilidad de lograr una buena comunicación médicopaciente mediante las TIC. ${ }^{9}$ Algunos estudios sugieren que pueden tener un efecto positivo en el manejo del asma, debido a que facilitan la comunicación médico-paciente y ayudan al paciente a aprender más sobre los cuidados de su enfermedad en el día a día. ${ }^{10}$ Sin embargo, para ese propósito es necesario conocer las tendencias de los pacientes: algunos eligen usar el correo electrónico y otros prefieren los mensajes de texto, si bien Facebook parece el medio social más apropiado para los más jóvenes. ${ }^{11}$

El objetivo de este estudio piloto fue evaluar la frecuencia y patrones de preferencia de las TIC en pacientes ecuatorianos con asma bronquial. Con los resultados se planea realizar un estudio para conocer la realidad latinoamericana. Los hallazgos podrían ayudar a mejorar el control personalizado de los pacientes con asma, la adherencia terapéutica y la relación médico-paciente.

\section{Métodos}

El presente estudio es observacional, de corte transversal, en el cual pacientes con asma respondieron de manera anónima un cuestionario que evaluaba la frecuencia de uso de TIC. Los pacientes procedían de instituciones públicas y privadas de las ciudades de Guayaquil, Quito y Portoviejo, Ecuador.

\section{Población}

Se incluyeron pacientes con edad $>12$ años y diagnóstico de asma bronquial de por lo menos un año de duración. Se excluyeron los pacientes que padecían alguna enfermedad psiquiátrica, trastornos para comprender el cuestionario o trastornos de la visión que les impidieran leerlo

\section{Procedimientos}

Los pacientes seleccionados fueron entrevistados usando el cuestionario de la Universidad de Michigan adaptado al español. ${ }^{11}$ Mediante 18 reactivos, con este cuestionario se evalúa información demográfica, uso de celulares y redes sociales, interés en el uso de dispositivos electrónicos y redes sociales para recibir información acerca del asma bronquial.

Se utilizó un método riguroso para traducir y adaptar el cuestionario al castellano: investigadores tradujeron las preguntas al español, luego esta versión fue traducida nuevamente al inglés por un tercer investigador, quien no conocía el cuestionario en su idioma original; cada reactivo fue evaluado por 30 individuos bilingües, no pertenecientes al estudio, quienes lo calificaron en una escala del 1 al 7 , siendo 7 la peor calificación para cada pregunta. Los reactivos con puntuación $>3$ fueron revisados, corregidos y volvieron a pasar por el mismo procedimiento. ${ }^{12}$

Se recolectó información demográfica de los pacientes como edad, sexo, raza, etnia e información sobre la medicación que utilizaban, duración de la enfermedad y nivel educativo. La escala para la cuantificación del uso de redes sociales (SMS, Facebook, Twitter, MySpace, correo electrónico, LinkedIn, YouTube, Skype) e internet constaba de las siguientes opciones: diariamente, al menos una vez por semana, al menos una vez por mes, menos de una vez por mes o nunca.

A los participantes también se les pidió que cuantificaran el interés en recibir y preguntar información acerca de los factores que intervienen con el control de la enfermedad mediante dispositivos electrónicos y redes sociales. El nivel de interés se midió con una escala cualitativa ordinal (alto, algo de interés, bajo y no interés).

En nuestro estudio se incorporó una nueva pregunta en el cuestionario, con la que se evaluó la frecuencia del uso de WhatsApp, debido a que esta aplicación fue lanzada en el 2013 y no está incluida en el estudio de Baptist et al. ${ }^{11}$ Se preguntó a los participantes si estaban interesados en recibir información o preguntar a su médico sobre su enfermedad usando esta aplicación. La respuesta obtenida en esta pregunta era de carácter dicotómico (sí o no).

Antes de recibir y realizar el cuestionario, los pacientes fueron informados acerca del propósito de este estudio y su papel en el mismo. Las encuestas fueron entregadas en la sala de espera antes o durante la consulta médica. Los pacientes podían responder el cuestionario solos o con la ayuda del personal de salud, previamente capacitado para esa tarea.

\section{Análisis estadístico}

Las respuestas para cada red social fueron dicotomizadas en al menos una vez por semana y menos de 
una vez por semana. La edad fue categorizada en tres grupos: 12-18 años (jóvenes), 19-40 años (adultos jóvenes) y mayores de 40 años (adultos). Además, el nivel educacional fue categorizado en educación primaria, secundaria y terciaria o de cuarto nivel. La variable "años con asma" fue dicotomizado en $\leq 5$ años y $>5$ años.

La prueba de chi cuadrada fue empleada para evaluar la asociación entre acceso a internet, poseer un teléfono celular o teléfono inteligente, edad y nivel educativo. La misma prueba estadística fue usada para evaluar la asociación entre edad, nivel educacional, sexo, tiempo de diagnóstico de asma y uso de al menos una vez a la semana de cada red social. Se empleó un análisis similar para determinar la asociación entre las categorías de edad, nivel educacional, sexo, años con asma y alto o algo de interés en recibir o preguntar información a través de cada tipo de red social.

Finalmente, fue efectuado análisis de regresión cruda para el uso e interés en comunicarse a través de las redes sociales. Asimismo, el análisis de regresión fue ajustado para edad y nivel educativo. Las categorías de referencias para la regresión fue edad $>40$ años y nivel educativo de ninguno/primaria. Todos los datos fueron analizados con el software SPSS $^{\circledR}$ versión 22.0 (SPSS Inc., Chicago, IL, EUA). Se utilizó un valor de $\mathrm{p}<0.05$ para establecer la significación estadística en todos los análisis.

\section{Resultados}

En total fueron recolectadas 222 encuestas. La media de edad fue de $45.6 \pm 17.4$ años y la mayoría de los participantes fue del sexo femenino $(89.2 \%)$ (Cuadro 1). La mayoría de los pacientes indicó tener teléfono celular $(87.5 \%)$ y en menor proporción, acceso a internet $(62.7 \%)$. Solo $46.1 \%$ de los participantes reportó tener un teléfono inteligente (Cuadro 2). En el análisis entre los grupos de edad, en los pacientes $>40$ años se observó la frecuencia más baja de acceso a internet $(47.5 \%)(p=0.002)$, así como de teléfonos inteligentes $(33.3 \%)(p=0.001)$ (Cuadro 2).

\section{Uso de TIC al menos una vez por semana}

En general, los SMS (mensajes de texto) fueron el medio electrónico más comúnmente utilizado $(64.6 \%)$, seguido de internet $(40.8 \%)$ y Facebook $(39.6 \%)$.
Los pacientes adultos reportaron las frecuencias más bajas de uso de Facebook, correo electrónico e internet $(\mathrm{p}<0.001)$. La frecuencia de uso de YouTube y Twitter en los adultos osciló en alrededor de $10 \%(\mathrm{p}<0.01)$. En contraste, el SMS fue el medio más usado por los adultos con asma $(\mathrm{p}<0.001)$ (Cuadro 3) (Figura 1).

Los pacientes de sexo masculino reportaron un mayor uso de Facebook, YouTube e Internet (83.3, 43.8 y $72.2 \%)$, ( $<<0.05)$ (Cuadro 4). Los pacientes con alto nivel educativo reportaron con mayor frecuencia el uso de redes sociales $(\mathrm{p}<0.01)$ (Cuadro 3).

Búsqueda de información relacionada con la enfermedad a través de TIC

Internet fue la fuente preferida para obtener información relacionada con la enfermedad (33.2\%). Los pacientes jóvenes y adultos jóvenes reportaron con mayor frecuencia el Internet como red preferida de búsqueda de información $(\mathrm{p}<0.001)$. En los adultos jóvenes, YouTube $y$ el correo electrónico fueron mayormente reportados como medios de búsqueda de información $(\mathrm{p}=0.005)($ Cuadro 3$)$.

Interés en recibir información sobre el asma a través de TIC

La mayoría de los pacientes indicó alto interés en recibir información a través de SMS (84.8\%), WhatsApp (39.4\%) y correo electrónico (33.0\%), sobre todo los jóvenes y adultos jóvenes (Cuadro 3).

Interés en preguntar al médico información sobre el asma a través de TIC

Los pacientes jóvenes y adultos jóvenes presentaron mayor interés en preguntar información sobre su enfermedad a través de Facebook y WhatsApp. En el grupo de los adultos jóvenes, el correo electrónico también obtuvo una frecuencia alta de interés (Cuadro 3).

\section{Sexo}

Los pacientes del sexo masculino mostraron mayor uso de Facebook, YouTube e internet (83.3, 43.8 y $72.2 \%)(p<0.05)$. Estos pacientes también reportaron un alto interés en recibir y preguntar información por medio del correo electrónico $(\mathrm{p}<0.05)$ y WhatsApp (p < 0.01) (Cuadro 4). 
Nivel de educación

Los pacientes con título universitario o de cuarto nivel de educación reportaron con mayor frecuencia acceso a internet y teléfonos inteligentes $(\mathrm{p}=0.001)$ (Cuadro 2), así como mayor uso de casi todas las redes sociales, con excepción de LinkedIn $(\mathrm{p}<0.01)$ (Cuadro 3).

En cuanto al interés de recibir y buscar información relacionada con la enfermedad, los pacientes con alto nivel educacional prefirieron casi todas las redes sociales y medios electrónicos $(\mathrm{p}<0.05)$, con excepción de LinkedIn y SMS.

\section{Análisis de regresión logística}

En los análisis de regresión ajustados para la edad (Cuadro 5), los adultos jóvenes con asma tuvieron casi cuatro veces más probabilidad de usar al menos una vez por semana SMS y Twitter, en comparación con los otros grupos de edad. Facebook, YouTube, correo electrónico e internet fueron más empleados por pacientes más jóvenes (OR, 10.6, 11.9 y 13.6, respectivamente).

Los pacientes de edad adulta tendieron a buscar información relacionada con la enfermedad con mayor probabilidad que los pacientes jóvenes (OR, 3.7), mientras que los adultos jóvenes tuvieron mayor probabilidad de estar interesados en recibir o preguntar información por correo electrónico (OR, 4.3 y 4.8 , respectivamente).

En el ajuste para el nivel educacional, WhatsApp fue la TIC en la que se observó mayor interés y probabilidad de uso (OR 9.8 and 8.5, respectivamente) ( $p<0.05)$, excepto en el grupo con baja escolaridad (Cuadro 6).

\section{Discusión}

Para mejorar el monitoreo y control de las enfermedades crónicas, diferentes estudios se han enfocado en instrumentos basados en TIC. Se ha reportado que $43.4 \%$ de los latinoamericanos está conectado a internet; ${ }^{13}$ en nuestra investigación se observó un porcentaje mayor (60\%). Además, más encuestados con mayor educación señalaron acceso a internet. Si bien en Latinoamérica se ha registrado un aumento en el uso de teléfonos inteligentes, ${ }^{14}$ en nuestro estudio menos de la mitad de los pacientes indicó tener un teléfono inteligente. Esta diferencia pone de manifiesto que probablemente el empleo de estrategias a través de estos dispositivos no sea adecuado en Ecuador. ${ }^{14}$

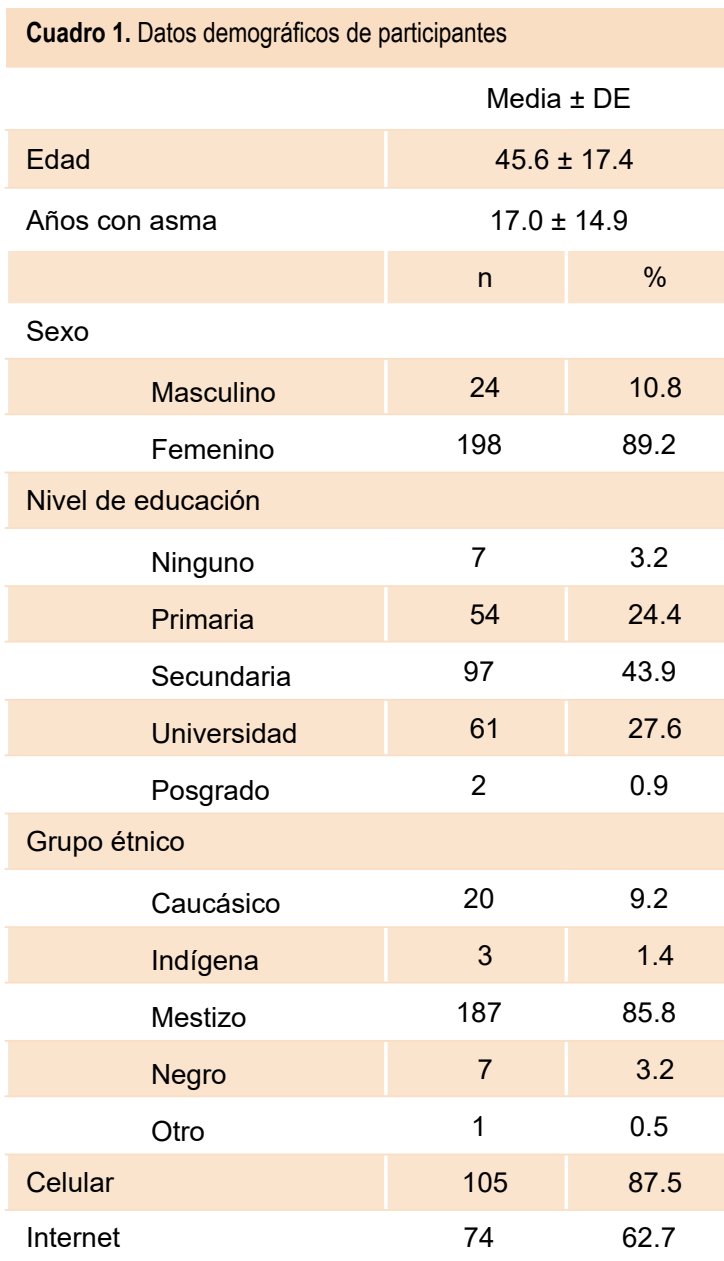

En términos generales, el mensaje de texto es la TIC más utilizada. Un metaanálisis concluyó que los mensajes de texto podrían aumentar al doble la adherencia a la medicación. ${ }^{15}$ Además, un estudio previo encontró que el correo electrónico y los mensajes de texto son los medios electrónicos más populares a través de los cuales los pacientes con asma desean recibir información y preguntar sobre su enfermedad a los médicos. ${ }^{11}$ En nuestro estudio identificamos resultados similares, ya que el mensaje de texto fue el más empleado, seguido del correo electrónico. Aun cuando sobresalió el interés por recibir y preguntar información relacionada con el asma por medio de WhatsApp, los resultados no se pueden comparar directamente con los obtenido en los otros recursos pues se empleó una escala de respuesta diferente (sí o no). 
Cuadro 2. Uso, búsqueda de información, interés en recibir información y preguntar al médico sobre asma a través de TIC, según grupos etarios
$\leq 18$ años
$>18$ a $\leq 40$ años
$>40$ años
$p$
$(\%)$
(chi cuadrada)
Total
(\%)
(\%)
(\%)

Usar por lo menos una vez a la semana:

SMS

69.2

82.9

53.0

0

64.6

Facebook

84.6

66.7

18.9

39.6

Twitter

25.0

34.3

2.9

0

19.2

YouTube

50.0

40.3

8.7

0.003

22.5

Correo electrónico

66.7

52.4

14.4

0

30.6

Internet

83.3

57.8

27.0

40.8

Linkedln

12.5

12.5

2.9

0

8.1

Skype

12.5

28.6

5.7

$0.294 \ddagger$

16.7

Buscar información sobre asma con:

\begin{tabular}{|l|c|c|c|c|c|}
\hline Internet & 46.2 & 50.7 & 21.7 & 0 & 33.2 \\
\hline Facebook & 8.3 & 8.8 & 4.6 & $0.369 \ddagger$ & 6.2 \\
\hline Twitter & 0 & 0 & 2.8 & $0.631 \ddagger$ & 1.7 \\
\hline YouTube & 9.1 & 21.7 & 5.4 & 0.005 & 11.0 \\
\hline Correo electrónico & 0 & 9.1 & 4.8 & $0.517 \ddagger$ & 6.0 \\
\hline
\end{tabular}

Estar altamente interesado en recibir información acerca de su enfermedad usando:

\begin{tabular}{|l|l|l|l|r|r|}
\hline SMS & 75.0 & 87.7 & 84.2 & 0.51 & 84.8 \\
\hline Facebook & 45.5 & 51.7 & 13.0 & 0 & 28.4 \\
\hline Twitter & 18.2 & 16.7 & 1.0 & $0.000 \ddagger$ & 7.5 \\
\hline Linkedln & 16.7 & 7.1 & 3.4 & $0.297 \ddagger$ & 6.3 \\
\hline Correo electrónico & 25.0 & 53.8 & 21.3 & 0 & 33.0 \\
\hline WhatsApp* & 69.2 & 68.3 & 18.6 & 0 & 39.4 \\
\hline
\end{tabular}

Estar altamente interesado en preguntar información acerca de su enfermedad usando:

\begin{tabular}{|c|c|c|c|c|c|}
\hline SMS & 75.0 & 89.4 & 83.3 & 0.337 & 84.9 \\
\hline Facebook & 45.5 & 50.0 & 13.1 & 0 & 27.7 \\
\hline Twitter & 9.1 & 17.0 & 1.0 & $0.001 \ddagger$ & 6.9 \\
\hline Linkedln & 16.7 & 3.7 & 3.7 & $0.446 \ddagger$ & 5.0 \\
\hline Correo electrónico & 25.0 & 57.8 & 22.2 & 0 & 34.8 \\
\hline WhatsApp* & 58.3 & 65.0 & 19.8 & 0 & 38.2 \\
\hline
\end{tabular}

Las diferencias en los valores entre los tres grupos etarios son significativas con nivel de significación de 0.05 .

†No se calculó chi cuadrada por el reducido tamaño de la muestra

$\ddagger$ Valor $p$ exacto de Fisher.

*La pregunta para la categoría WhatsApp fue dicotomizada como Sí/No. 
Chérrez-Ojeda I et al. Preferencias en el uso de TIC en personas con asma

Cuadro 3. Características de usuarios frecuentes de TIC ( $\geq 1 /$ semana) que mostraron mucho/algo de interés en recibir información y preguntar al médico acerca de asma

Interés en recibir información según Interés en preguntar al médico tipo de TIC

por TIC

Variable
RM
IC $95 \%$
RM
IC $95 \%$

SMS ( $n=199)$

Edad†

\begin{tabular}{|l|c|c|c|c|}
\hline$<18$ años & 0.6 & $0.1-2.3$ & 0.6 & $0.1-2.4$ \\
\hline $18-40$ años & 1.3 & $0.5-3.3$ & 1.7 & $0.7-4.2$ \\
\hline
\end{tabular}

Nivel educativoł

\begin{tabular}{|c|c|c|c|c|}
\hline Secundaria & 2.6 & $1.03-6.4$ & 2.6 & $1.1-6.4$ \\
\hline Nivel superior/cuarto nivel & 2.7 & $0.9-7.9$ & 5.0 & $1.5-16.3$ \\
\hline Uso semanal de SMS* & 3.4 & 1.5-7.9 & 5.2 & $2.2-12.3$ \\
\hline
\end{tabular}

Edad

\begin{tabular}{|l|l|l|l|r|}
\hline$<18$ años & 5.6 & $1.5-20.9$ & 5.5 & $1.5-20.7$ \\
\hline $18-40$ años & 7.2 & $3.3-15.6$ & 6.6 & $3.0-14.5$ \\
\hline
\end{tabular}

Nivel educativo

\begin{tabular}{|c|c|c|c|c|}
\hline Secundaria & 3.5 & $1.2-10.2$ & 2.9 & $1.0-8.3$ \\
\hline Nivel superior/cuarto nivel & 8.3 & $2.8-24.5$ & 8.6 & $2.9-25.6$ \\
\hline Uso semanal de Facebook & 29.3 & $11.5-74.6$ & 26.0 & $10.2-66.1$ \\
\hline
\end{tabular}

Correo electrónico $(n=187)$

Edad

\begin{tabular}{|l|l|l|l|l|}
\hline$<18$ años & 1.2 & $0.3-4.9$ & 1.2 & $0.3-4.6$ \\
\hline $18-40$ años & 4.3 & $2.2-8.4$ & 4.8 & $2.4-9.4$
\end{tabular}

Nivel educativo

\begin{tabular}{|c|c|c|c|c|}
\hline Secundaria & 5.0 & $1.6-15.5$ & 3.5 & $1.2-10.1$ \\
\hline Nivel superior/cuarto nivel & 19.4 & $6.1-61.7$ & 16.7 & $5.7-49.1$ \\
\hline nal de correo electrónico & 21.5 & $9.4-49.1$ & 23.2 & $10.0-53.9$ \\
\hline
\end{tabular}

Las regresiones fueron ajustadas para las variables edad y nivel educativo. Los análisis de regresión para uso semanal de TIC fueron elaborados por separado y no ajustados. Todos los valores mostrados son significativos a nivel de significancia de 0.05 . RM, razón de momios; IC, intervalo de confianza.

†La categoría de referencia de edad es $>40$ años.

$\ddagger$ La categoría de referencia del nivel educativo es ninguno/primaria.

"La categoría de referencia de uso de TIC es "menos que semanal". 


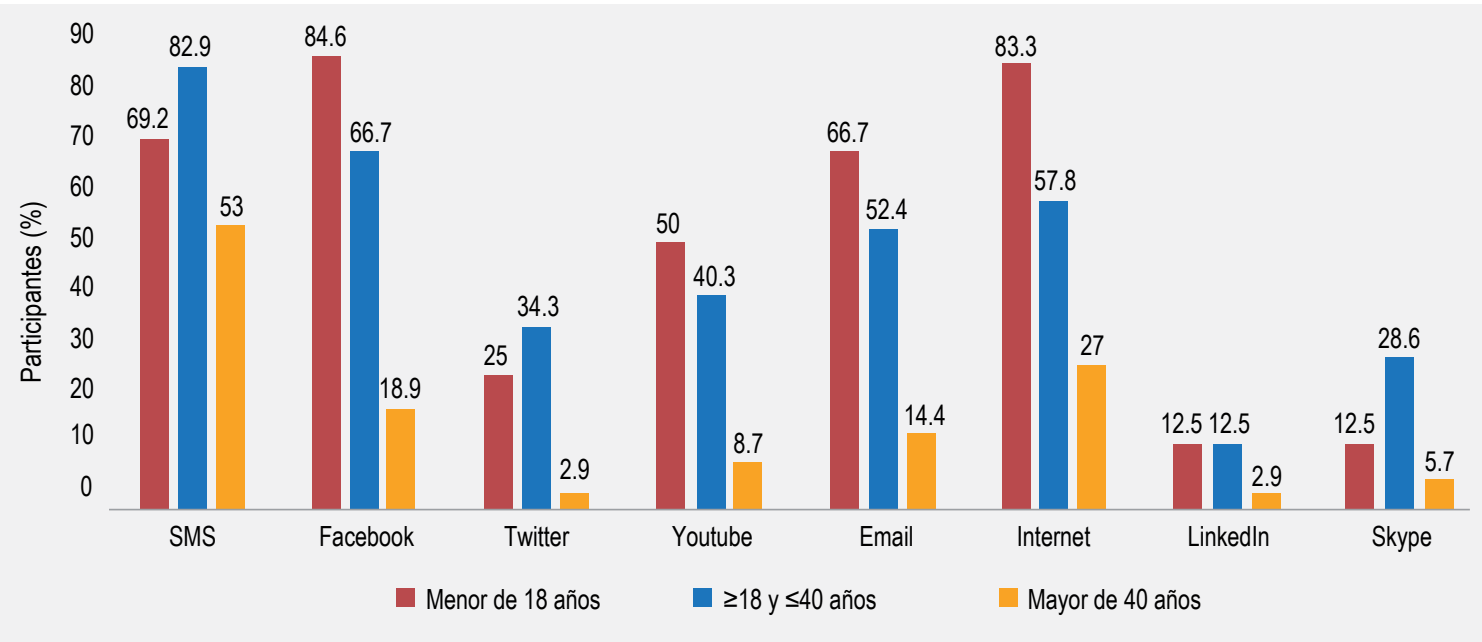

Figura 1. Uso de al menos una vez por semana de cualquier tecnología de información y comunicación.

Nuestros hallazgos sugieren que tanto el mensaje de texto y el correo electrónico son las herramientas preferidas para recibir y preguntar al médico sobre el asma bronquial, independientemente de la edad. Existen algunas razones prácticas para el uso frecuente de mensajes de texto: es más barato que un mensaje de voz, el mensaje puede ser enviado y recibido, aun cuando la persona tenga apagado su celular. ${ }^{16}$ Por otro lado, si el personal de salud desea enviar alguna información, esta puede ser mejor comunicada, debido a la privacidad que ofrece este recurso. ${ }^{17}$

Los pacientes jóvenes con asma han mostrado más interés en recibir información y preguntar a su médico utilizando medios sociales como Facebook. ${ }^{18}$ Por otro lado, se ha reportado que los hispanos creen que los proveedores de salud deberían usar Facebook y Twitter para compartir informa-

Cuadro 4. Características de usuarios de WhatsApp que mostraron mucho/algo de interés en recibir por ese medio información y preguntar al médico acerca del asma

\begin{tabular}{|c|c|c|c|c|}
\hline \multirow[b]{2}{*}{ Variable } & \multicolumn{2}{|c|}{$\begin{array}{l}\text { Interés en recibir información } \\
\text { por WhatsApp }\end{array}$} & \multicolumn{2}{|c|}{$\begin{array}{l}\text { Interés en preguntar al médico } \\
\text { por WhatsApp }\end{array}$} \\
\hline & RM & IC $95 \%$ & $\mathrm{RM}$ & IC $95 \%$ \\
\hline \multicolumn{5}{|l|}{ Edad $†$} \\
\hline$<18$ años & 9.8 & $2.7-35.3$ & 5.7 & $1.6-19.7$ \\
\hline $18-40$ años & 9.4 & 4.5-19.7 & 7.5 & $3.6-15.5$ \\
\hline \multicolumn{5}{|l|}{ Nivel educativoł } \\
\hline Secundaria & 2.3 & $0.9-5.6$ & 1.9 & $0.8-4.7$ \\
\hline Nivel superior/cuarto nivel & 9.8 & $3.8-25.2$ & 8.5 & $3.3-22.0$ \\
\hline
\end{tabular}


Chérrez-Ojeda I et al. Preferencias en el uso de TIC en personas con asma

Cuadro 5. Características de usuarios frecuentes de TIC ( $\geq 1$ vez/semana) que mostraron mucho/algo de interés en recibir información y preguntar al médico acerca de asma

Interés en recibir información según tipo de TIC
Interés en preguntar al médico por TIC

Variable

\begin{tabular}{|l|l|l|l}
\hline RM & IC $95 \%$ & RM & IC $95 \%$ \\
\hline
\end{tabular}

SMS $(n=199)$

Edad $\dagger$

\begin{tabular}{|l|l|l|l|l|}
\hline$<18$ años & 0.6 & $0.1-2.3$ & 0.6 & $0.1-2.4$ \\
\hline $18-40$ años & 1.3 & $0.5-3.3$ & 1.7 & $0.7-4.2$ \\
\hline
\end{tabular}

Nivel educativoł

\begin{tabular}{|c|c|c|c|c|}
\hline Secundaria & 2.6 & $1.03-6.4$ & 2.6 & $1.1-6.4$ \\
\hline Nivel superior/cuarto nivel & 2.7 & $0.9-7.9$ & 5.0 & $1.5-16.3$ \\
\hline Uso semanal de SMS* & 3.4 & $1.5-7.9$ & 5.2 & $2.2-12.3$ \\
\hline
\end{tabular}

Facebook ( $n=188)$

Edad

\begin{tabular}{|l|l|l|l|r|}
\hline$<18$ años & 5.6 & $1.5-20.9$ & 5.5 & $1.5-20.7$ \\
\hline $18-40$ años & 7.2 & $3.3-15.6$ & 6.6 & $3.0-14.5$ \\
\hline
\end{tabular}

Nivel educativo

\begin{tabular}{|c|c|c|c|c|}
\hline Secundaria & 3.5 & IC $95 \%$ & 2.9 & $1.0-8.3$ \\
\hline Nivel superior/cuarto nivel & 8.3 & $2.8-24.5$ & 8.6 & $2.9-25.6$ \\
\hline Uso semanal de Facebook & 29.3 & $11.5-74.6$ & 26.0 & $10.2-66.1$ \\
\hline
\end{tabular}

Correo electrónico $(n=187)$

Edad

\begin{tabular}{|l|l|l|l|r|}
\hline$<18$ años & 1.2 & $0.3-4.9$ & 1.2 & $0.3-4.6$ \\
\hline $18-40$ años & 4.3 & $2.2-8.4$ & 4.8 & $2.4-9.4$ \\
\hline
\end{tabular}

Nivel educativo

\begin{tabular}{|c|c|c|c|c|}
\hline Secundaria & 5.0 & $1.6-15.5$ & 3.5 & $1.2-10.1$ \\
\hline Nivel superior/cuarto nivel & 19.4 & $6.1-61.7$ & 16.7 & $5.7-49.1$ \\
\hline Uso semanal de e-mail & 21.5 & $9.4-49.1$ & 23.2 & $10.0-53.9$ \\
\hline
\end{tabular}

Las regresiones fueron ajustadas para las variables edad y nivel educativo. Los análisis de regresión para uso semanal de TIC fueron elaborados por separado (no ajustados). Todos los valores son significativos a nivel de significación de 0.05 . RM, razón de momios; IC, intervalo de confianza.

†La categoría de referencia de la edad es $>40$ años.

łLa categoría de referencia del nivel educativo es ninguno/primaria.

*La categoría de referencia del uso de TIC es "menos que semanal". 
ción sobre salud, ${ }^{19}$ por lo tanto, estas TIC son apropiadas para el desarrollo en Ecuador de estrategias para mejorar el automanejo del asma bronquial.

WhatsApp es una TIC que permite intercambiar textos, imágenes, videos y mensajes de audio. En Latinoamérica, dos tercios de los usuarios de internet ocupan su tiempo "whatsapiando", en contraste con las personas que viven en Norteamérica, donde es poco conocido y usado. ${ }^{20}$

Nuestros resultados sugieren que WhatsApp es una herramienta apropiada para recibir y preguntar al médico sobre el asma bronquial, en especial los jóvenes están más dispuestos a usar la aplicación, así como los de mayor nivel educativo. Una de las explicaciones para el amplio uso de esta TIC podría ser la disponibilidad de paquetes de servicios de telefonía móvil donde tanto WhatsApp como Facebook pueden ser usados en forma ilimitada sin costo alguno.

En nuestros hallazgos resalta el mayor uso de TIC por parte de los pacientes masculinos, aun cuando en nuestro estudio la mayoría de los participantes fue del sexo femenino, en contraste con hallazgos previos donde las mujeres fueron las que más emplearon las TIC. ${ }^{11}$

Los resultados obtenidos en este estudio piloto pueden tener implicaciones para el desarrollo de futuras intervenciones en pacientes con asma bron- quial basadas en las TIC. A partir de ellos se infiere que ciertos medios sociales pueden ser de mejor utilidad que otros en los jóvenes con asma.

Las nuevas tecnologías pueden hacer llegar información a pacientes que viven en zonas urbanas o rurales de difícil acceso y a los pacientes con asma mal controlada. Estas tecnologías podrían lograr un avance e incremento de los cuidados de la enfermedad. Mediante las TIC, muchas estrategias de manejo de asma podrían personalizarse mediante herramientas interactivas con un contenido rico en gráficos, basada en los datos individuales de los participantes. Además, se podrían enviar videos sobre el uso correcto de los inhaladores, lo cual contribuiría en mejorar su utilización y disminuir uno de los factores de mal control del asma. Los medios electrónicos también podrían involucrar a profesionales de la salud para mejorar la educación de los pacientes con asma.

Sin embargo, aún falta analizar cómo nuestros resultados puedan tener una utilidad práctica en la vida real. Primero será necesario desarrollar, en conjunto con los ingenieros de software, una aplicación personalizada y amigable adecuada a las necesidades del paciente. Por otro lado, se debe replicar el estudio en otros países de Latinoamérica para comparar las coincidencias y diferencias en el comportamiento de los pacientes con asma.

\begin{tabular}{|c|c|c|c|c|}
\hline \multirow[t]{2}{*}{ Variable } & \multicolumn{2}{|c|}{$\begin{array}{l}\text { Interés en recibir información por } \\
\text { WhatsApp }\end{array}$} & \multicolumn{2}{|c|}{$\begin{array}{l}\text { Interés en preguntar al médico por } \\
\text { WhatsApp }\end{array}$} \\
\hline & $\mathrm{RM}$ & IC $95 \%$ & $\mathrm{RM}$ & IC $95 \%$ \\
\hline \multicolumn{5}{|l|}{ Edad† } \\
\hline$<18$ años & 9.8 & $2.7-35.3$ & 5.7 & $1.6-19.7$ \\
\hline $18-40$ años & 9.4 & $4.5-19.7$ & 7.5 & $3.6-15.5$ \\
\hline \multicolumn{5}{|l|}{ Nivel educativoł } \\
\hline Secundaria & 2.3 & $0.9-5.6$ & $1.90 .8-4.7$ & \\
\hline Nivel superior/cuarto nivel & 9.8 & $3.8-25.2$ & 8.5 & $3.3-22.0$ \\
\hline \multicolumn{5}{|c|}{$\begin{array}{l}\text { Las regresiones fueron ajustadas para las variables edad y nivel educativo. Todos los valores mostrados son significativos con el nivel de signifi- } \\
\text { cación de } 0.05 \text {. RM, razón de momios; IC, intervalo de confianza. } \\
\text { †La categoría de referencia de la edad es }>40 \text { años. } \\
\text { †La categoría de referencia del nivel educativo es Ninguno/primaria. }\end{array}$} \\
\hline
\end{tabular}


Nuestro estudio presenta algunas limitaciones. Primero, es un estudio de corte transversal y la relación causa-efecto no puede ser demostrada; se requieren ensayos clínicos aleatorizados para hacerlo. Por otro lado, el cuestionario Michigan no ha sido validado y los resultados podrían tener conclusiones inexactas y sesgadas. Además, no conocemos si los pacientes usan las TIC con mayor frecuencia durante las crisis asmáticas, cuando tienen comorbilidades o para automedicarse, preguntas que deberán contestarse en futuras investigaciones.

Una fortaleza de nuestro estudio es el tamaño de la muestra, grande a pesar de tratarse de un estudio piloto. En esta investigación participaron pacientes de uno y otro sexo, con diferentes edades, nivel de educación y lugar de residencia.

Con los resultados se pueden desarrollar plataformas personalizadas accesibles a los pacientes con asma, que pueden ser difundidas en los sitios web de las sociedades de alergia o neumología, con lo que se lograría que los pacientes cuenten con información respaldada científicamente.

Son necesarias futuras investigaciones para probar si la aplicación de las TIC logra mejorar la adherencia al tratamiento, el automanejo del asma y la calidad de vida, así como la disminución de las exacerbaciones, de las visitas a las salas de emergencias y las consultas médicas. Estos resultados podrán in- ducir a las organizaciones gubernamentales a implementar estrategias nacionales para ayudar a mejorar la atención en Ecuador de los pacientes con asma.

Finalmente, podemos decir que WhatsApp es una alternativa de comunicación muy interesante, altamente sugerida por los participantes de este estudio, especialmente por los jóvenes.

\section{Conclusión}

En nuestro estudio piloto se demostró que los mensajes de texto y el correo electrónico pueden ser utilizados por pacientes de todas las edades, sin embargo, los otros medios sociales, como Facebook y WhatsApp, serían útiles en los pacientes jóvenes. Esta información puede servir para desarrollar estrategias personalizadas de TIC conforme a los requerimientos de cada paciente. Serán necesarias futuras investigaciones para examinar los beneficios reales de las TIC.

El uso generalizado de las redes sociales abre nuevas posibilidades para la relación médico-paciente y la comunicación en ambas direcciones.

\section{Agradecimientos}

Al programa MECOR, en especial a Sonia Buist y Ana Menezes. Del mismo modo, nuestro agradecimiento a las doctoras Linda Vera y Gabriela Martinetti, así como a todos los miembros de Respiralab, por su apoyo para realizar este estudio.

\section{Referencias}

1. Croisant S. Epidemiology of Asthma: Prevalence and burden of disease. En: Brasier AR, editor. Heterogeneity in asthma. EUA: Springer; 2014.

2. Masoli M, Fabian D, Holt S, Beasley R, Global Initiative for Asthma Program. The global burden of asthma: executive summary of the GINA Dissemination Committee report. Allergy. 2004;59(5):469-478. DOI: http://dx.doi.org/10.1111/j.1398-9995.2004.00526.x

3. Gold LS, Montealegre F, Allen-Ramey FC, Jardim J, Smith N, Sansores R, et al. Level of asthma control and healthcare utilization in Latin America. Allergy. 2013;68(11):1463-1466. DOI: http://dx.doi.org/10.1111/all.12237.

4. Bender BG, Bender SE. Patient-identified barriers to asthma treatment adherence: responses to interviews, focus groups, and questionnaires. Immunol Allergy Clin North Am. 2005;25(1):107-130. DOI: http://dx.doi.org/10.1016/j.iac.2004.09.005

5. Nathan RA, Thompson PJ, Price D, Fabbri LM, Salvi S, González-Díaz S, et al. Taking aim at asthma around the world: global results of the asthma insight and management survey in the Asia-Pacific region, Latin America, Europe, Canada, and the United States. J Allergy Clin Immunol Pract. 2015;3(5):734.e5742.e5. DOI: http://dx.doi.org/10.1016/j.jaip.2015.04.013

6. Chérrez-Ojeda I, Calderón JC, Mori J, Colombaro D, Braido F, Soria E, et al. Patient-physician relationship in the management of asthma: Multicentric approach in Latin America. J Asthma. 2016;53(7):751-760. DOI: http://dx.doi.org/10.3109/02770903.2016.1145691 
7. Jiménez-Pernett J, García-Gutiérrez JF, Martín-Jiménez JL, Bermúdez-Tamayo C. Tendencias en el uso de Internet como fuente de información sobre salud. UOC Papers. 2007(4):44-50. Disponible en: http:// www.redalyc.org/articulo.oa?id=79000411

8. Perron BE, Taylor HO, Glass JE, Margerum-Leys J. Information and communication technologies in social work. Adv Soc Work. 2010;11(2):67-81.

9. Buse DC, Lipton RB. Facilitating communication with patients for improved migraine outcomes. Curr Pain Headache Rep. 2008;12(3):230-236.

10. Lombardi C, Passalacqua G, Canonica GW. The WEB-based Asthma Control: an intriguing connection or a dangerous hazard? Asthma Res Pract. 2015;1(1):15. DOI: http://dx.doi.org/10.1186/s40733-0150017-z

11. BaptistAP, Thompson M, Grossman KS, Mohammed L, SyA, Sanders GM. Social media, text messaging, and email-preferences of asthma patients between 12 and 40 years old. J Asthma. 2011;48(8):824-830. DOI: http://dx.doi.org/10.3109/02770903.2011.608460

12. Sperber AD. Translation and validation of study instruments for cross-cultural research. Gastroenterology. 2004;126 Suppl 1:S124-S128. DOI: http://dx.doi.org/10.1053/j.gastro.2003.10.016

13. Naciones Unidas/CEPAL. [Sitio web]. Estado de la banda ancha en América Latina y el Caribe 2016. Serie Documentos de Proyectos núm. 710; 2016. Disponible en: http://repositorio.cepal.org/ handle/11362/40528

14. Pew Research Center. [Sitio web]. Smith A. U. S. smartphone use in 2015. [Actualizado 2015 Abr 1]. http://www.pewinternet.org/2015/04/01/us-smartphone-use-in-2015/

15. Thakkar J, Kurup R, Laba TL, Santo K, Thiagalingam A, Rodgers A, et al. Mobile telephone text messaging for medication adherence in chronic disease: A meta-analysis. JAMA Intern Med. 2016;176(3):340-349. DOI: http://dx.doi.org/10.1001/jamainternmed.2015.7667

16. Kaplan WA. Can the ubiquitous power of mobile phones be used to improve health outcomes in developing countries? Global Health. 2006;2(1):9. DOI: http://dx.doi.org/10.1186/1744-8603-2-9

17. Chretien KC, Kind T. Social media and clinical care: Ethical, professional, and social implications. 2013;127(13):1413-1421. DOI: http://dx.doi.org/10.1161/CIRCULATIONAHA.112.128017

18. Chou W-YS, Hunt YM, Beckjord EB, Moser RP, Hesse BW. Social media use in the United States: Implications for health communication. J Med Internet Res. 2009;11(4):e48. DOI: http://dx.doi. org/10.2196/jmir.1249

19. Hanson CL, West J, Thackeray R, Barnes MD, Downey J. Understanding and predicting social media use among community health center patients: A cross-sectional survey. J Med Internet Res. 2014;16(11):e270. DOI: http://dx.doi.org/10.2196/jmir.3373

20. GlobalWeblndex. [Sitio web]. Young K. WhatsApp usage highest in LatAm. GlobalWeblndex. The trends to watch in 2017. Reino Unido: GlobalWeblndex; 2016. https://blog.globalwebindex.net/chart-of-theday/whatsapp-usage-highest-in-latam/ 